\title{
Improving Adherence to Guidelines for the Diagnosis and Management of Pelvic Inflammatory Disease: A Systematic Review
}

\author{
Bette Liu, ${ }^{1}$ Basil Donovan, ${ }^{1,2}$ Jane S. Hocking, ${ }^{3}$ Janet Knox, $^{1}$ \\ Bronwyn Silver, ${ }^{1,4}$ and Rebecca Guy ${ }^{1}$ \\ ${ }^{1}$ The Kirby Institute, University of New South Wales, Sydney, NSW 2052, Australia \\ ${ }^{2}$ Sydney Sexual Health Centre, Sydney Hospital, Sydney, NSW 2000, Australia \\ ${ }^{3}$ Centre for Women's Health, Gender and Society, Melbourne School of Population Health, University of Melbourne, \\ Melbourne, VIC 3010, Australia \\ ${ }^{4}$ Menzies School of Health Research, Alice Springs, NT 0870, Australia
}

Correspondence should be addressed to Bette Liu, bliu@kirby.unsw.edu.au

Received 14 May 2012; Accepted 19 July 2012

Academic Editor: Thomas Cherpes

Copyright ( $) 2012$ Bette Liu et al. This is an open access article distributed under the Creative Commons Attribution License, which permits unrestricted use, distribution, and reproduction in any medium, provided the original work is properly cited.

Background. Evidence suggests adherence to clinical guidelines for pelvic inflammatory disease (PID) diagnosis and management is suboptimal. We systematically reviewed the literature for studies describing strategies to improve the adherence to PID clinical guidelines. Methods. The databases MEDLINE and EMBASE, and reference lists of review articles were searched from January 2000 to April 2012. Only studies with a control group were included. Results. An interrupted time-series study and two randomised controlled trials (RCTs) were included. The interrupted time-series found that following a multifaceted patient and practitioner intervention (practice protocol, provision of antibiotics on-site, written instructions for patients, and active followup), more patients received the recommended antibiotics and attended for followup. One RCT found a patient video on PID self-care did not improve medication compliance and followup. Another RCT found an abbreviated PID treatment guideline for healthpractitioners improved their management of PID in hypothetical case scenarios but not their diagnosis of PID. Conclusion. There is limited research on what strategies can improve practitioner and patient adherence to PID diagnosis and management guidelines. Interventions that make managing PID more convenient, such as summary guidelines and provision of treatment on-site, appear to lead to better adherence but further empirical evidence is necessary.

\section{Introduction}

Pelvic inflammatory disease (PID), particularly mild-to-moderate disease, can be difficult to diagnose as the symptoms and signs are often nonspecific, and there is no gold standard that confirms the diagnosis [1-4]. Because the consequences of untreated PID may be severe, current clinical guidelines recommend that practitioners have a high index of suspicion for the diagnosis of PID and a low threshold for empirical treatment [5-8]. Clinical guidelines outline the symptoms and signs of PID and likely causative organisms in different patient populations, appropriate diagnostic tests, and empirical broad-spectrum antibiotics that are available and appropriate, given local considerations regarding antibiotic resistance and whether the PID was thought to be sexually acquired, postpartum or postprocedural. Guidelines also advise on outpatient or inpatient treatment, management of sexual partners, and appropriate follow-up of patients to assess the response to treatment.

Internationally, research suggests that the diagnosis and management of PID could be improved. In a survey of 200 UK general practitioners, less than half were able to name two symptoms and two signs of PID or correctly name an antibiotic regimen [9], and a recent study of over 2000 women with PID seen in general practice found that only $34 \%$ were treated with recommended antibiotic regimens 
and 54\% were tested for chlamydia [10]. Audits of US emergency department records have found that less than half of the patients seen with a diagnosis of PID are prescribed antibiotics according to the Centers for Disease Control and Prevention guidelines [11-13]. An audit in an Australian sexual health centre suggested substantial variation in PID diagnoses between practitioners [14], and another audit found that in a high chlamydia prevalence area, symptoms and signs of PID were commonly recorded, but the majority of women were not presumptively diagnosed with PID, nor managed in accordance with the local guidelines [15].

Given there are current evidence-based clinical guidelines for the diagnosis and management of PID but substantial data suggesting poor adherence to these guidelines, this paper aims to examine what strategies may improve adherence to PID diagnosis and management guidelines.

\section{Methods}

This paper was undertaken according to the Preferred Reporting Items for Systematic Reviews and Meta-Analyses (PRISMA) guidelines [16].

2.1. Search Strategy. The electronic databases MEDLINE and EMBASE (from January 2000 to April 2012) were searched. Reference lists of review articles were also examined for relevant articles. The search terms and strategy used comprised the following:

(i) "pelvic inflammatory disease" or "PID" or "salpingitis" or "adnexitis"

(ii) "diagnosis" or "management."

The reference lists obtained were limited to the English language and humans. The titles of all articles were reviewed and if relevant, the abstract examined. If the abstract appeared to meet the inclusion criteria, the full text article was obtained and was reviewed for inclusion.

2.2. Inclusion Criteria. We included all studies that examined the effect of an intervention to improve practitioner or patient adherence to diagnostic or management guidelines for PID. Only studies that had a control or comparison group were included.

2.3. Data Extraction. For each study the following data items were extracted: study type, setting and population, number of participants, interventions, outcomes, and findings. As the nature of studies included in this paper varied substantially, no attempt was made to combine the data in a metaanalysis. Instead the studies are reviewed individually, and their application to the improvement of PID diagnosis and management is discussed.

\section{Results}

The screening of studies is outlined in Figure 1. After excluding duplicates, there were 2314 titles identified from the electronic databases and of these, 203 abstracts were reviewed. Eighteen full-text articles were obtained, and

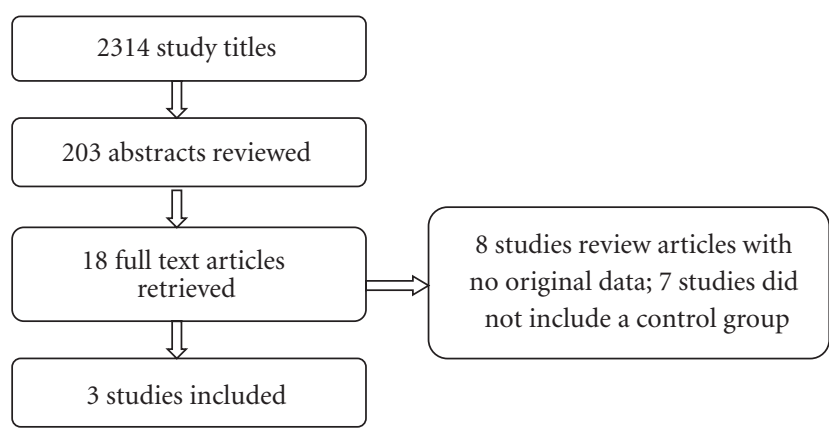

Figure 1: Screening of studies for inclusion in review.

of these, three studies met the inclusion criteria and are outlined in Table 1 [17-19]. Of the full-text articles that were excluded, eight were review articles rather than original research and the other seven did not measure the effect of an intervention to improve adherence.

The three included studies examined a variety of interventions aimed at the patient, the practitioner, or both. Two studies were randomised controlled trials (RCTs) and one was an interrupted time series study. All three studies were conducted in the US between 2001 and 2008 and were aimed at paediatric and adolescent populations, or practitioners caring for these populations. The studies were based in hospital and outpatient facilities; one was based at a single hospital, the other two involved multiple centres (Table 1). For the studies involving interventions with patients, the populations were young (mean age 16 or 17 years) and predominantly African American, while for studies involving practitioners, they were practicing US paediatric emergency physicians.

\subsection{Description of Individual Studies}

3.1.1. Multifaceted Approach. The interrupted time-series study by Trent et al. [19] aimed to assess if using a multifaceted approach in a single academic paediatric outpatient setting improved the management of mild-to-moderate PID. The intervention targeted both practitioners and their patients. Practitioners were provided with a PID-treatment algorithm, clinical practice guidelines, and training to follow the PID care protocol. The patients were given the initial empirical treatment at the site as well as the full 14-day course of antibiotics to take home. They were also given written discharge instructions and were followed by a telephone call at 24-48 hours and at 2 weeks.

The intervention was evaluated by extraction of data from administrative and medical records in the year prior to the intervention and then compared to data recorded and extracted over a nine-month period during the intervention. Logs were used to record physician use of the clinical practice guidelines and medications during the intervention period. The medical records for 56 patients diagnosed with PID and treated as an outpatient before the intervention and 71 patients following the intervention were compared. Patients diagnosed before and after the intervention were of similar age and race, and had similar insurance status and 
TABLE 1: Summary of controlled studies to improve adherence to diagnosis and management guidelines for PID.

\begin{tabular}{|c|c|c|c|c|c|c|}
\hline Study ID & Study type & Setting/Year & Target population & $N^{*}$ & Intervention & Outcomes \\
\hline $\begin{array}{l}\text { Trent et } \\
\text { al. [19] }\end{array}$ & $\begin{array}{l}\text { Interrupted } \\
\text { time series }\end{array}$ & $\begin{array}{l}\text { A single paediatric } \\
\text { outpatient department } \\
\text { in the US, 2001-2003 }\end{array}$ & $\begin{array}{l}\text { Patients and } \\
\text { practitioners }\end{array}$ & 127 & $\begin{array}{l}\text { Multilevel intervention: } \\
\text { practitioner treatment algorithm } \\
\text { and practice guideline, full } \\
\text { 14-day course of antibiotics for } \\
\text { patients and written discharge } \\
\text { instructions, telephone } \\
\text { follow-up at } 24-48 \text { hrs and } 2 \\
\text { weeks. }\end{array}$ & $\begin{array}{l}\text { Patients receiving } \\
\text { appropriate medication, } \\
\text { return for follow-up. }\end{array}$ \\
\hline $\begin{array}{l}\text { Trent et } \\
\text { al. [17] }\end{array}$ & RCT & $\begin{array}{l}5 \text { hospital emergency } \\
\text { departments or } \\
\text { paediatric and } \\
\text { adolescent medicine } \\
\text { clinics in a large urban } \\
\text { US centre, } \sim 2007\end{array}$ & $\begin{array}{l}\text { 15-21-year-old } \\
\text { women diagnosed } \\
\text { with outpatient } \\
\text { treatable PID in }\end{array}$ & 121 & $\begin{array}{l}\text { 6-minute video using health } \\
\text { belief model to acknowledge } \\
\text { barriers and benefits to PID } \\
\text { self-care in addition to } \\
\text { standardised care. }\end{array}$ & $\begin{array}{l}\text { Medication completion, } \\
\text { temporary sexual } \\
\text { abstinence during the } \\
\text { 14-day treatment period, } \\
\text { partner notification, } \\
\text { partner treatment, return } \\
\text { for 72-hour follow-up. }\end{array}$ \\
\hline $\begin{array}{l}\text { Balamuth } \\
\text { et al. [18] }\end{array}$ & RCT & $\begin{array}{l}\text { Members of the section } \\
\text { on Emergency Medicine } \\
\text { of the American } \\
\text { Academy of Paediatrics, } \\
\text { US, } 2008\end{array}$ & $\begin{array}{l}\text { Physicians working } \\
\text { in paediatric } \\
\text { emergency } \\
\text { departments }\end{array}$ & 237 & $\begin{array}{l}\text { Use of a PID-treatment summary } \\
\text { sheet versus the full CDC } \\
\text { PID-treatment guidelines. }\end{array}$ & $\begin{array}{l}\text { Correct completion of a } \\
\text { multiple choice survey on } \\
\text { PID diagnosis, treatment } \\
\text { and follow-up. }\end{array}$ \\
\hline
\end{tabular}

${ }^{*} \mathrm{~N}$ is the total number of population including both intervention and control groups.

positivity rates for gonorrhoea and chlamydia. During the post-intervention period, uptake of the intervention varied; $65 \%$ of practitioners used the clinical practice guidelines and $52 \%$ distributed the patient information sheet; $88 \%$ of patients received the 14-day course of antibiotics; $50 \%$ were contactable by telephone within $24-48$ hours and $38 \%$ were contactable at 2 weeks.

Comparing the management of PID diagnosed in the period before and after the intervention, testing for gonorrhoea and chlamydia were similarly high ( $98 \%$ before versus $100 \%$ after) but requesting wet-film microscopy of vaginal discharge increased (3\% versus $38 \%$ ). The proportion of patients receiving the recommended antibiotics increased following the intervention ( $62 \%$ versus $91 \%$, adjusted OR 8.4 (95\% CI 2.6-26.8); $P<0.001$ ), and so did the proportion who attended for follow-up within 72 hours (10\% versus $43 \%$, statistical test of significance not provided).

\subsubsection{Randomised Controlled Trial of a Patient Educational}

Video. Trent et al. subsequently conducted an RCT of an educational video aimed at improving PID self-management in adolescents diagnosed with mild-to-moderate PID [17]. The researchers recruited 126 adolescents who presented to one of five clinical sites. All patients were managed using the multifactorial approach described in Trent et al. [19], that is, practitioners were given the PID treatment algorithm and clinical practice guidelines, and the treatment site provided patients with the full 14-day course of empirical antibiotics. In addition to this routine management, the patients were randomised to an intervention that involved watching a sixminute video or the control group which did not have the video. The video aimed to have patients better acknowledge the barriers and benefits of PID self-care.
Interviews were conducted before and after the intervention on all participants in order to compare baseline characteristics and evaluate outcomes between the two groups. The baseline interview included information on sociodemographics, sexual, and reproductive history and the follow-up interview was conducted following two weeks of treatment to assess outcomes. The baseline characteristics of the study population were similar between groups. None of the outcomes examined at two-week follow-up, including completing the course of antibiotics (intervention versus control: $66 \%$ versus $66 \%$ resp., OR 0.99 (95\% CI $0.38-$ 2.57)), follow-up visit within 72 hours (32\% versus $16 \%$, OR 2.55 (95\% CI 0.82-7.89)), abstention from intercourse (78\% versus $89 \%$, OR 0.46 (95\% CI $0.12-1.75)$ ), partner notification ( $88 \%$ versus $92 \%$, OR 0.64 (95\% CI $0.13-$ $3.10)$ ), and partner treatment ( $71 \%$ versus $53 \%$, OR 2.16 (95\% CI $0.82-5.72)$ ) was found to differ statistically in the unadjusted analysis although the authors performed adjusted analyses and found that increased partner treatment became significant (OR 3.10 (95\% CI 3.10 (1.03-9.39)).

\subsubsection{Randomised Controlled Trial of an Abbreviated PID Treatment Summary Sheet. Balamuth et al. [18] conducted an RCT aimed at improving practitioner diagnosis and man- agement of PID using an abbreviated PID treatment sum- mary sheet versus the full 2006 Centres for Disease Control and Prevention (CDC) Sexually Transmitted Disease man- agement guidelines [20]. The 237 recruited emergency pae- diatricians were randomised to a either a weblink to the 2006 CDC treatment guidelines or a one-page PID treatment sum- mary sheet that had been developed by the authors based on the most salient points from the CDC guidelines. The inter- vention was evaluated by a self-completed online multiple}


choice survey assessing their diagnosis and treatment of PID. The characteristics of practitioners were generally similar between comparison groups. More practitioners reported using the summary sheet than the weblink (79\% versus $50 \%$ resp.). Significant differences were also found between groups for the proportion of practitioners who chose the correct antibiotics (summary sheet versus weblink: 97\% versus 61\%, OR 19.4 (95\% CI 6.6-76.9)) and who made the correct follow-up recommendations (76\% versus $32 \%$, OR 6.6 (95\% CI 3.6-12.2)). The proportion making the correct diagnosis (45\% versus 50\%, OR 0.82 (95\% CI 0.49 1.40) ) and choosing the correct admission criteria ( $87 \%$ versus 90\%, OR 1.3 (95\% CI 0.6-2.9)) did not differ between groups.

\section{Discussion}

4.1. Methodological Limitations. Two studies included in this review were RCTs and therefore of a higher quality for assessing interventions than the observational study. However, all studies still had methodological issues that limit their interpretation or generalisability. Overall the studies were relatively small, and conducted on adolescent patient groups in hospital or outpatient settings. Due to the nature of the studies and the interventions, allocation of the intervention was not concealed to either participants or the outcome assessors.

Specifically, the RCT by Trent et al. [17] which did not find any benefit to PID management with the video intervention in the intention to treat analysis, was limited by the loss to follow-up. The outcomes of interest assessed at 2-week follow-up were missing for 39\% of participants. The RCT by Balamuth was limited primarily by the hypothetical nature of the study outcome and the generalisability of the study findings. Rather than actual clinical diagnoses of PID and treatment of real patients, the outcomes were hypothetical responses to survey questions. Only 35\% of physicians invited to complete the study were included and it is unknown if those who took part differed from those who did not take part. Also, randomisation to the intervention occurred before recruitment to the study (at the point of email invitation), and while baseline characteristics of study participants were similar between groups, smaller numbers of study participants were allocated to the intervention group than the control (109 versus 128). The 2006 study by Trent found a significant difference in some outcomes although the study design meant that differences between the populations measured before and after the intervention that could potentially bias the findings, could not be adequately accounted for. Also, while the absolute numbers of PID diagnoses made before and after the intervention ( 56 versus 71) may suggest that the intervention improved the diagnosis rate of PID, the lack of denominators and hence underlying rate of PID diagnosis at the study site meant that this kind of interpretation was not possible. Similarly, as the intervention was multifaceted, the contribution made by various aspects of the intervention to the improved patient antibiotic adherence and attendance at follow-up could not be distinguished.
4.2. Generalisability of Findings. In many settings the majority of PID cases, especially mild-to-moderate disease, are managed by general practitioners or primary care physicians rather than in hospital settings by specialists [21]. Therefore applying the findings from these three small US studies to general recommendations on the diagnosis and management of PID is difficult. While Balamuth found that abbreviated summary PID clinical guidelines may improve practitioner treatment of PID, such a strategy should be tested in clinical settings with patients. Trent et al.'s earlier multifaceted intervention to improve PID diagnosis and management was an observational study rather than a trial. Therefore while some components, such as the provision of the 14-day course of antibiotics at presentation and written discharge instructions may be innovative and feasible, they may require further study in a trial setting, and should consider issues such as the socioeconomic status of the patient population before being recommended for widespread implementation.

4.3. Future Directions of Research to Improve PID Diagnosis and Management. While they were not included in this paper because they did not assess improved compliance as an outcome, some recent RCTs of antibiotics for PID have compared simplified regimens such as once-daily dosing using moxifloxacin instead of ofloxacin $[22,23]$ or onceweekly dosing using azithromycin instead of doxycycline [24] to more conventional treatments under the premise that simplified regimens will result in greater patient adherence to treatment [25]. These studies have found that for uncomplicated PID management, the simplified regimens have had similar or better clinical and microbial cure rates than the conventional treatment, although none of these studies have examined longer term sequelae. Also as PID, particularly mild and moderate disease is recognised as being difficult to diagnose due to the nonspecific symptoms and signs and the range of possible differential diagnoses, there is a need for noninvasive but specific diagnostic tests and clinical case definitions to make the identification of PID simpler for health practitioners.

In Australia, as part of a trial of population screening for chlamydia (http://www.accept.org.au/), an evaluation of a PID education package delivered to general practitioners is currently underway [26]. The package involves reading materials and a DVD on PID diagnosis and management. Practitioner diagnosis rates, antibiotic treatments prescribed and practitioner knowledge surveys measured before and after the intervention will be used to assess the effectiveness of the educational package. A recent review also found that health practitioners may not follow clinical guidelines for a variety of reasons including both knowledge and experience but also beliefs and values [27]. The review suggested that guidelines should not only summarize evidence from clinical trials, but also provide data on the cost-benefit and patient preferences to make them more applicable to clinical practice.

\section{Conclusions and Recommendations}

This paper found that there is little research in the area of improving practitioner and patient adherence to PID 
diagnosis and management guidelines. Only three studies were identified, and because of the study settings and limitations in their methodology, caution should be taken in recommending their widespread application to clinical practice. Based on the findings from this systematic review we suggest that further studies, particularly in primary care settings where diagnosis and management of PID has been found to be suboptimal, should be conducted. Studies that compare any one of the following interventions to current practice should be investigated to determine if they improve the diagnosis and management of PID: abbreviated practitioner clinical management guidelines, provision of the full course of antibiotic treatment to the patient at presentation, simplified antibiotic regimens, and written instructions for patients.

\section{Acknowledgments}

B. Liu, B. Donovan, J. S. Hocking, and R. Guy are supported by research fellowships from the Australian National Health and Medical Research Council.

\section{References}

[1] I. Simms, F. Warburton, and L. Weström, "Diagnosis of pelvic inflammatory disease: time for a rethink," Sexually Transmitted Infections, vol. 79, no. 6, pp. 491-494, 2003.

[2] P. E. Munday, "Pelvic inflammatory disease-an evidencebased approach to diagnosis," Journal of Infection, vol. 40, no. 1, pp. 31-41, 2000.

[3] C. E. Blenning, J. Muench, and D. Z. Judkins, "Which tests are the most useful for diagnosing PID?" Journal of Family Practice, vol. 56, no. 3, pp. 216-220, 2007.

[4] O. Jaiyeoba and D. E. Soper, "A practical approach to the diagnosis of pelvic inflammatory disease," Infectious Diseases in Obstetrics \& Gynecology, vol. 2011, Article ID 753037, 6 pages, 2011.

[5] British Association for Sexual Health and HIV, "United Kingdom national guideline for the management of pelvic inflammatory disease," 2005, http://www.bashh.org/documents/118/ 118.pdf.

[6] Centres for Disease Control and Prevention, "Sexually transmitted diseases treatment guidelines, 2010," Morbidity and Mortality Weekly Report, vol. 59, pp. 63-67, 2010.

[7] J. Ross, P. Judlin, and L. Nilas, "European guideline for the management of pelvic inflammatory disease," International Journal of STD and AIDS, vol. 18, no. 10, pp. 662-666, 2007.

[8] Sexual Health Society of Victoria, "National management guidelines for sexually transmissible infections," 2008, http:// www.mshc.org.au/Guidelines/NationalManagementGuidelinesForSTIs/tabid/278/Default.aspx.

[9] I. Simms, M. R. Vickers, J. Stephenson, P. A. Rogers, and A. Nicoll, "National assessment of PID diagnosis, treatment and management in general practice: england and Wales," International Journal of STD and AIDS, vol. 11, no. 7, pp. 440-444, 2000.

[10] A. Nicholson, G. Rait, T. Murray-Thomas, G. Hughes, C. H. Mercer, and J. Cassell, "Management of first-episode pelvic inflammatory disease in primary care: results from a large UK primary care database," British Journal of General Practice, vol. 60, article e395, no. 579, 2010.
[11] B. G. Kane, L. C. Degutis, H. K. Sayward, and G. D’Onofrio, "Compliance with the centers for disease control and prevention recommendations for the diagnosis and treatment of sexually transmitted diseases," Academic Emergency Medicine, vol. 11, no. 4, pp. 371-377, 2004.

[12] K. R. Beckmann, M. D. Melzer-Lange, and M. H. Gorelick, "Emergency Department Management of Sexually Transmitted Infections in US Adolescents: results from the National Hospital Ambulatory Medical Care Survey," Annals of Emergency Medicine, vol. 43, no. 3, pp. 333-338, 2004.

[13] T. Y. Shih, C. A. Gaydos, R. E. Rothman, and Y. H. Hsieh, "Poor provider adherence to the centers for disease control and prevention treatment guidelines in US emergency department visits with a diagnosis of pelvic inflammatory disease," Sexually Transmitted Diseases, vol. 38, no. 4, pp. 299-305, 2011.

[14] A. Doxanakis, R. D. Hayes, M. Y. Chen et al., "Missing pelvic inflammatory disease? Substantial differences in the rate at which doctors diagnose PID," Sexually Transmitted Infections, vol. 84, no. 7, pp. 518-523, 2008.

[15] B. Silver, A. Rumbold, K. Smith et al., "How is pelvic inflammatory disease being managed in remote central Australian primary health care centres," in Proceedings of the Australasian Sexual Health Congress, Sydney, Australia, 2010.

[16] http://www.prisma-statement.org/.

[17] M. Trent, S. E. Chung, M. Burke, A. Walker, and J. M. Ellen, "Results of a randomized controlled trial of a brief behavioral intervention for pelvic inflammatory disease in adolescents," Journal of Pediatric and Adolescent Gynecology, vol. 23, no. 2, pp. 96-101, 2010.

[18] F. Balamuth, H. Zhao, and C. Mollen, "Toward improving the diagnosis and the treatment of adolescent pelvic inflammatory disease in emergency departments: results of a brief, educational intervention," Pediatric Emergency Care, vol. 26, no. 2, pp. 85-92, 2010.

[19] M. Trent, S. L. Judy, J. M. Ellen, and A. Walker, "Use of an institutional intervention to improve quality of care for adolescents treated in pediatric ambulatory settings for pelvic inflammatory disease," Journal of Adolescent Health, vol. 39, no. 1, pp. 50-56, 2006.

[20] Centres for Disease Control and Prevention, "Sexually transmitted diseases treatment guidelines 2006," Morbidity and Mortality Weekly, vol. 55, article RR-11, pp. 1-94, 2006.

[21] M. Y. Chen, Y. Pan, H. Britt, and B. Donovan, "Trends in clinical encounters for pelvic inflammatory disease and epididymitis in a national sample of Australian general practices," International Journal of STD and AIDS, vol. 17, no. 6, pp. 384-386, 2006.

[22] J. D. C. Ross, H. S. Cronjé, T. Paszkowski et al., "Moxifloxacin versus ofloxacin plus metronidazole in uncomplicated pelvic inflammatory disease: results of a multicentre, double blind, randomised trial," Sexually Transmitted Infections, vol. 82, no. 6, pp. 446-451, 2006.

[23] P. Judlin, Q. Liao, Z. Liu, P. Reimnitz, B. Hampel, and P. Arvis, "Efficacy and safety of moxifloxacin in uncomplicated pelvic inflammatory disease: the MONALISA study," An International Journal of Obstetrics and Gynaecology, vol. 117, no. 12, pp. 1475-1484, 2010.

[24] R. F. Savaris, L. M. Teixeira, T. G. Torres, M. I. A. Edelweiss, J. Moncada, and J. Schachter, "Comparing ceftriaxone plus azithromycin or doxycycline for pelvic inflammatory disease: a randomized controlled trial," Obstetrics and Gynecology, vol. 110, no. 1, pp. 53-60, 2007. 
[25] J. Dunbar-Jacob, S. M. Sereika, S. M. Foley, D. C. Bass, and R. B. Ness, "Adherence to oral therapies in pelvic inflammatory disease," Journal of Women's Health, vol. 13, no. 3, pp. 285-291, 2004.

[26] J. Hocking and N. Low, "ACCEPt (Australian Chlamydia Control Effectiveness Pilot): design of a chlamydia testing cluster randomised controlled trial in general practice," in Proceedings of the International Symposium on Human Chlamydial Infections, Salzburg, Austria, 2010.

[27] I. Baiardini, F. Braido, M. Bonini, E. Compalati, and G. W. Canonica, "Why do doctors and patients not follow guidelines?" Current Opinion in Allergy and Clinical Immunology, vol. 9, no. 3, pp. 228-233, 2009. 


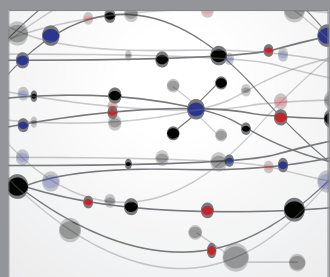

The Scientific World Journal
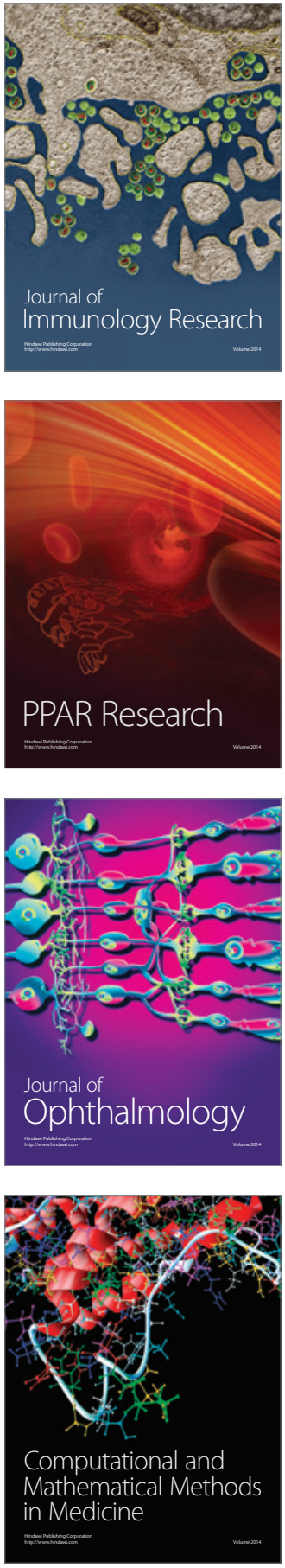

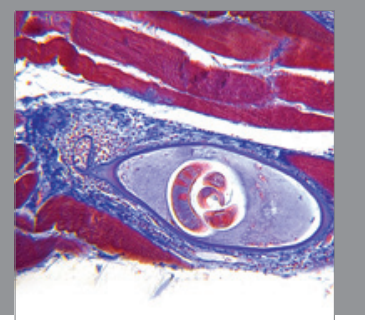

Gastroenterology

Research and Practice
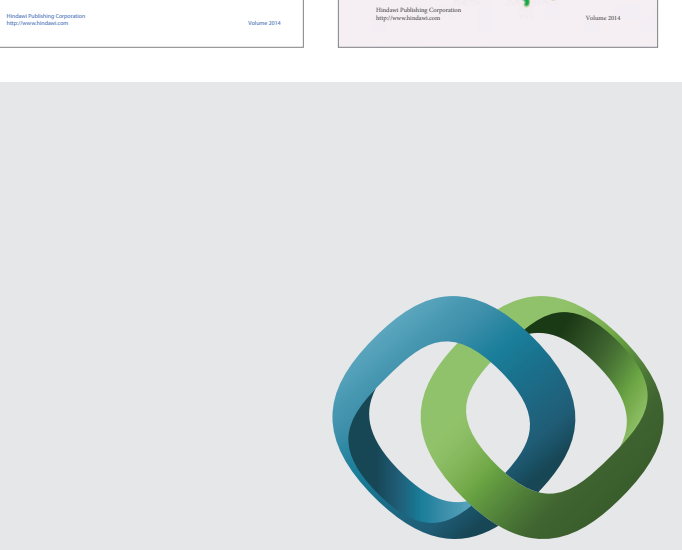

\section{Hindawi}

Submit your manuscripts at

http://www.hindawi.com
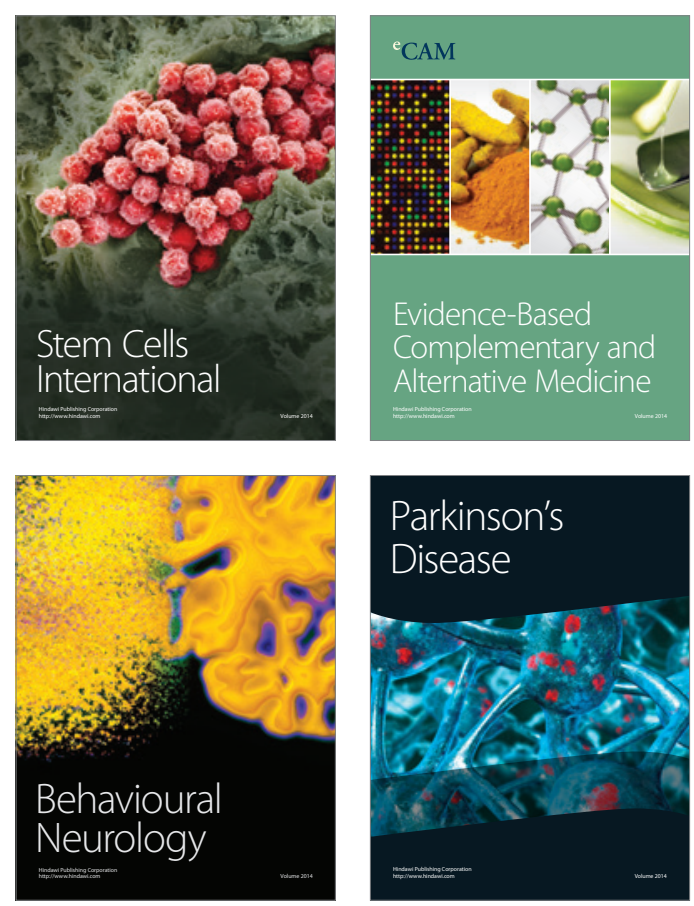

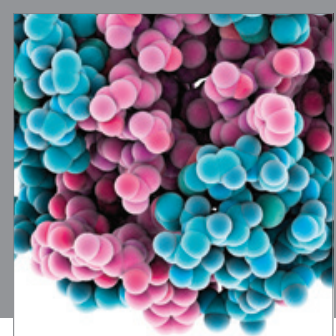

Journal of
Diabetes Research

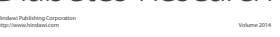

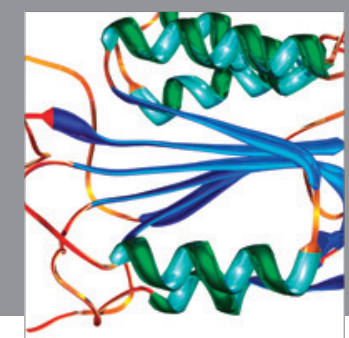

Disease Markers
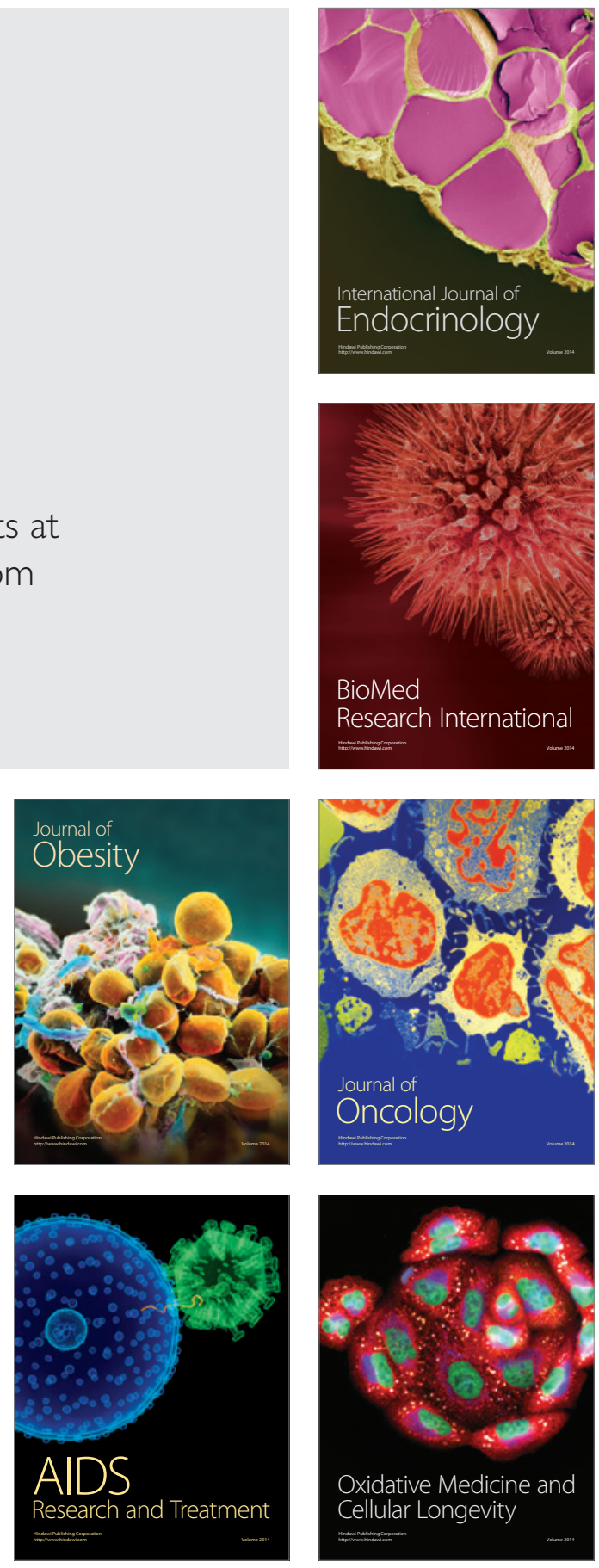